\title{
THE COHOMOLOGY OF THE COMPLEX PROJECTIVE STIEFEL MANIFOLD
}

\author{
BY \\ CARLOS ALFREDO RUIZ(')
}

0 . Let $U_{n}$ be the unitary group of order $n$. We have inclusions

$$
\cdots \supset U_{n} \supset U_{n-1} \supset \cdots U_{1} \approx S^{1} \text {. }
$$

We denote by $W_{n, n-m}$ the complex Stiefel manifold $W_{n, n-m}=U_{n} / U_{m}$ and by $Y_{n, n-m}^{\prime}$ the complex projective Stiefel manifold which we defined as follows: $S^{1}$, regarded as the set of complex numbers of module 1 , acts by multiplication on $U_{n}$. This action being compatible with the above inclusions defines an action of $S^{1}$ on $W_{n, n-m}$ and we define $Y_{n, n-m}^{\prime}$ as the set of orbits.

In particular we have

$$
\begin{aligned}
W_{n, 1} & =U_{n} / U_{n-1} \approx S^{2 n-1}, \\
W_{n, n} & =U_{n}, \\
Y_{n, n}^{\prime} & =P U_{n}, \text { the projective unitary group. }
\end{aligned}
$$

In this paper we compute $\left(H^{*} Y_{n, k}^{\prime}\right)$. Baum and Browder [1] have obtained our result in the special case $n=p^{r}, m=0$.

In order to state our main result we need some notation: Let $\omega$ be the generator of $H^{*}\left(C P^{\infty}\right)=\mathbf{Z}[\omega]$ and $z_{i}$ the generators of $H^{*}\left(W_{n, k}\right)=\bigwedge\left(z_{m+1}, \ldots, z_{n}\right)$. Let $b_{i}=$ G.C.D. $\left(\left(C_{n, m+1}, \ldots, C_{n, i}\right)\right)$. Finally in $\S 1$, we will show there is a fibration

$$
W_{n, n-m} \underset{i}{\longrightarrow} Y_{n, n-m} \underset{\pi}{\longrightarrow} C P^{\infty}
$$

with $Y_{n, n-m}$ of the same homotopy type as $Y_{n, n-m}^{\prime}$. Then our main theorem is

THEOREM A.

$$
H^{*}\left(Y_{n, n-m}\right)=\mathrm{Z}[y] / I \otimes \wedge\left(v_{m+2}, \ldots, v_{n}\right), \text { where } \pi^{*} \omega=y
$$

$i^{*} v_{i}=\left(b_{i-1} / b_{i}\right) z_{i}$ and $I$ is the ideal generated by $b_{i} y^{i}, i=m+1, \ldots, n$.

In $\$ 1$ we compute $H^{*}\left(Y_{n, k} ; \mathbf{Z}_{p}\right)$ and $H^{*}\left(Y_{n, k} ; \mathbf{Q}\right)$ following the Gitler and Handel proof for real case [3]. In $\S 2$ we determine $\operatorname{Ker} \pi^{*}$ and $\operatorname{Im} i^{*}$. In $\S 3$ we show that this information is enough to determine all relevant Bockstein homomorphisms

Received by the editors June 13, 1969.

( ${ }^{1}$ Supported by Consejo Nacional de Investigaciones Cientificas y Tecnicas de la Republica Argentina.

Copyright (C) 1970, American Mathematical Society 
and compute the Bockstein spectral sequence for every prime $p$. This determines completely $H^{*}\left(Y_{n, k}\right)$.

We wish to thank Professors M. Mahowald and S. Gitler for their helpful suggestions and comments.

1. First we construct a space $Y_{n, k}$ of the same homotopy type as $Y_{n, k}^{\prime}$.

Observe that we have a principal bundle $S^{1} \rightarrow W_{n, n-m} \rightarrow Y_{n, n-m}^{\prime}$.

Let $\xi$ be the associate line bundle.

Proposition 1. $n \xi=\xi \oplus \xi \oplus \cdots \oplus \xi$ has $n-m$ C-linearly independent sections and it is the universal bundle for bundles $n \zeta$ having $n-m$ C-linearly independent sections, where $\zeta$ is a line bundle.

Proof. The proof is similar to the real case given in [3].

The inclusion $U_{m} \subset U_{n}$ gives rise to a fibration of classifying spaces

$$
W_{n, n-m} \underset{i_{1}}{\longrightarrow} B U_{m} \underset{\pi_{1}}{\longrightarrow} B U_{n}
$$

and the transgression satisfies

$$
\tau z_{i}=\sigma_{i} \text { where } \sigma_{i} \text { is the universal Chern class. }
$$

Now let $\gamma$ be the canonical line bundle over $C P^{\infty}$ and let $f_{n}$ be the classifying map of $n \gamma$ and let

$$
W_{n, n-m} \underset{i}{\longrightarrow} Y_{n, n-m} \underset{\pi}{\longrightarrow} C P^{\infty}
$$

be the fibration induced from (A) by $f_{n}$.

It is easy to see that the bundle induced by $f_{n} \pi$ is a universal bundle for the $n$-plane bundles satisfying the conditions of Proposition 1. Thus we have

Proposition 2. $Y_{n, n-m}$ and $Y_{n, n-m}^{\prime}$ have the same homotopy type.

From (1) and naturality of Chern classes and transgression

$$
\tau z_{i}=C_{n, i} \omega^{i} .
$$

If $x \in H^{*}(E)$ we denote by $\bar{x}$ (resp. $\left.\tilde{x}\right)$ its image in $H^{*}\left(E ; \mathbf{Z}_{p}\right)\left(\right.$ resp. $H^{*}(E ; \mathbf{Q})$ ).

Let $N(p)$ be the smallest $i$ such that $m+1 \leqq i<n$ and $C_{n, i}$ is not zero, $\bmod p$. The following theorem is similar to [3, Theorem 1.6].

THEOREM 3.

$$
\begin{aligned}
H^{*}\left(Y_{n, n-m} ; \mathbf{Z}_{p}\right) & =\mathbf{Z}_{p}[\bar{y}] /\left[y^{N(p)}\right] \otimes \wedge\left(\bar{x}_{m+1} \cdots \bar{x}_{N(p)} \cdots \bar{x}_{n}\right), \\
H^{*}\left(Y_{n, n-m} ; \mathbf{Q}\right) & =\mathbf{Q}[y] /\left[y^{m+1}\right] \otimes \wedge\left(\tilde{x}_{m+2} \cdots \tilde{x}_{n}\right),
\end{aligned}
$$

where $p$ is a prime and $\mathbf{Q}$ is the set of rational numbers; $i^{*}\left(\bar{x}_{i}\right)=\bar{z}_{i} ; \pi^{*}(\bar{\omega})=\bar{y}$; and $i^{*}\left(\tilde{x}_{i}\right)=\tilde{z}_{i}, \pi^{*}(\tilde{\omega})=\tilde{y}$. 
Proof. Let $K$ be a field, $K=\mathrm{Z}_{p}$ or $K=\mathbf{Q}$ and let $N$ be the smallest $i$ such that $m+1 \leqq i<n$ and $C_{n, i}$ is not zero in $K$.

In the spectral sequence of (B) with coefficients $K$ we have

$$
E_{2}=K[\omega] \otimes \wedge\left(z_{m+1} \cdots z_{n}\right) .
$$

By (2) $d_{r} z_{i}=0, r<2 i, d_{2 i} z_{i}=\tau z_{i}=C_{n, i} \omega^{i}$, then $d_{r}=0, r<2 N$ and then $E_{2}=E_{2 N}$; but

$$
d_{2 N} z_{N}=C_{n, N} \omega^{N} \neq 0 \text {, }
$$

thus, $z_{N}$ does not survive in $E_{2 N+1}$ and the image of the ideal [ $\left.\omega^{N}\right]$ in $E_{2 N+1}$ is 0 . Moreover, $E_{2 N+1}$ is still a tensor product:

$$
E_{2 \dot{N}+1}^{*, 0} \otimes E_{2 \dot{N}^{*}+1}^{0 *}=E_{2 \dot{N}+1}^{* * *}
$$

Now the following transgressions are zero (thus all the following differentials are zero), hence

$$
E_{\infty}=E_{2 N+1}=K[\omega] /\left[\omega^{N}\right] \otimes \bigwedge\left(z_{m+1} \cdots \hat{z}_{N} \cdots z_{n}\right) .
$$

The theorem now follows from Borel [2].

Corollary 4. $H^{*}\left(Y_{n, n-m}\right)$ has $p$-torsion if and only if $p$ divides $C_{n, m+1}$.

2. In this section we obtain the first results about $H^{*}\left(Y_{n, k}\right)$. The key is Proposition 5 below on $\operatorname{Ker} \pi^{*}$. In Corollary 7 we pick some elements in $H^{*}\left(Y_{n, k}\right)$ and using them we choose new generators for $H^{*}\left(Y_{n, k} ; \mathbf{Z}_{p}\right)$ and $H^{*}\left(Y_{n, k} ; \mathbf{Q}\right)\left((7),\left(7^{\prime}\right)\right)$.

Proposition 5.

$$
\operatorname{Ker} \pi^{*}=\left[b_{m+1} \omega^{m+1} ; \ldots, b_{n} \omega^{n}\right]
$$

Corollary 6.

$$
\operatorname{Ker} \bar{\pi}^{*}=\left[b_{m+1} \omega^{m+2} ; \ldots, b_{n} \omega^{n+1}\right] .
$$

Let $c_{i}=b_{i-1} / b_{i}, i=m+2, \ldots, n$.

Corollary 7.

$$
\begin{gathered}
T^{2 m+1} / \operatorname{Im} i^{*}=\mathrm{Z}, \quad T^{q} / \operatorname{Im} i^{*}=0, \quad q \geqq 2 n, \\
T^{2 i-1} / \operatorname{Im} i^{*}=\mathrm{Z}_{c i}, \quad m+2 \leqq i \leqq n .
\end{gathered}
$$

Thus, there exist elements $v_{i}$ in $H^{*}\left(Y_{n, n-m}\right)$ such that $i^{*} v_{i}=c_{i} z_{i}, m+2 \leqq i \leqq n$.

Before we give the proof we recall some facts about transgression in the spectral sequence of a fibration.

We use the transgression in (B) as defined in the following diagram, $\tau=$ $j^{*} \circ\left(\bar{\pi}^{*}\right)^{-1} \circ \delta$

$$
\begin{array}{r}
H^{*}\left(Y_{n, n-m}\right) \underset{i^{*}}{\longrightarrow} H^{*}\left(W_{n, n-m}\right) \underset{\delta}{\longrightarrow} H^{*}\left(Y_{n, n-m}, W_{n, n-m}\right) \longrightarrow H^{*}\left(Y_{n, n-m}\right) \\
H^{*}\left(C P^{\infty},{ }^{*}\right) \underset{j^{*}}{\longrightarrow}
\end{array} H^{*}\left(C P^{\infty}\right) .
$$




We recall that in the spectral sequence of $(\mathrm{B}) E_{2}^{0.2 i-1} \approx H^{2 i-1}\left(W_{n, n-m}\right)$. This isomorphism carries the subgroup $E_{2 i}^{0.2 i-1}$ onto $T^{2 i-1}$, the subgroup of transgressive elements. Also $E_{2}^{2 i, 0} \approx H^{2 i}\left(C P^{\infty}\right)$ and this isomorphism induces the isomorphism of quotient groups $E_{2 i}^{2 i, 0} \approx H^{2 i}\left(C P^{\infty}\right) / \operatorname{Ker}^{2 i} \bar{\pi}^{*}$. Moreover, via these isomorphisms, $\tau$ corresponds to $d_{2 i}^{0.2 i-1}$ and

$$
\operatorname{Im} d_{2 i}^{0,2 i-1} \approx \operatorname{Ker}^{2 i} \pi^{*} / \operatorname{Ker}^{2 i} \bar{\pi}^{*} \subset H^{2 i}\left(C P^{\infty}\right) / \operatorname{Ker}^{2 i} \bar{\pi}^{*} .
$$

Finally, $\tau$ induces an isomorphism

$$
T^{a-1} / \operatorname{Im} i^{*} \approx \operatorname{Ker}^{q} \pi^{*} / \operatorname{Ker}^{q} \bar{\pi}^{*} .
$$

Consider the diagram:

(C)

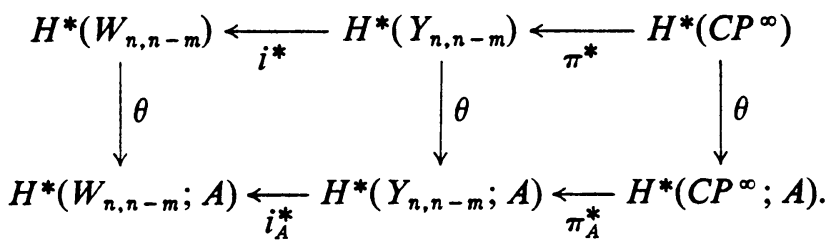

If $A=\mathbf{Z}_{p}$, then Theorem 2 and $i^{*} \circ \pi^{*}=0$ yield

$$
\operatorname{Ker} i_{\mathbf{z}_{p}}^{*}=[\bar{y}]
$$$$
\operatorname{Ker} \pi_{\mathbf{Z}_{p}}^{*}=\left[\omega^{N(p)}\right] \text {. }
$$

If $A=\mathbf{Q}$, we have

$$
\begin{aligned}
\operatorname{Ker} i_{\mathbf{Q}}^{*} & =[\tilde{y}], \\
\operatorname{Ker} \pi_{\mathbf{Q}}^{*} & =\left[\tilde{\boldsymbol{\omega}}^{m+1}\right] .
\end{aligned}
$$

Proof of Proposition 5. The spectral sequence of (B) is trivial through $E_{2 m+2}$. Thus $E_{2 m+2}^{2 m+2,0} \approx H^{2 m+2}\left(C P^{\infty}\right)$ and $\operatorname{Ker}^{q} \bar{\pi}^{*}=0, q \leqq 2 m+2$.

From (2) and (3) we obtain

$$
\operatorname{Ker}^{q} \pi^{*}=0, \quad q<2 m+2 \text { and } \operatorname{Ker}^{2 m+2} \pi^{*}=\left[C_{n, m+1} \omega^{m+1}\right]^{2 m+2} .
$$

Applying (2) repeatedly we have

$$
\left[b_{m+1} \omega^{m+1}, \ldots, b_{n} \omega^{n}\right] \subset \operatorname{Ker} \pi^{*} \subset H^{*}\left(C P^{\infty}\right) .
$$

For the other inclusion, put $b_{i}=a p^{r}$, where $p$ does not divide $a$. We use diagram (C) with $A=\mathbf{Z}_{p} r$, if $p^{s} c \omega^{i}$ belongs to Ker $\pi^{*}, s<r$ and $c$ divides $a$, then $p^{s} c \theta(\omega)^{i}$ belongs to $\operatorname{Ker} \pi_{\mathbf{Z}_{p}}^{*}$ but it is not 0 because $c$ is not a divisor of 0 in $\mathbf{Z}_{p} r$. On the other hand in the spectral sequence of (B) with coefficients $\mathbf{Z}_{p} r$

$$
\tau z_{k}=C_{n, k} \omega^{k}=0, \quad k<i,
$$

because $p^{r}$ divides $C_{n, k}$ for those $k$. Thus, the spectral sequence is trivial through $2 i$ and then $\operatorname{Ker}^{2 i} \pi_{\mathbf{Z}_{p} r}^{*}=0$. 
Proof of Corollary 6. Follows from (3) and Proposition 5.

Proof of Corollary 7. First part follows from (4), second part follows trivially from first part.

The elements $v_{i}$ are not unique, we will choose a fixed set of such elements arbitrarily.

In diagram (C) with $\mathbf{Z}_{p}=A$, we have:

(i) if $p$ does not divide $c_{i}, \theta v_{i}=c_{i} \bar{x}_{i}+u_{i}, m+2 \leqq i \leqq n$ where $u_{i} \in \operatorname{Ker} i_{\mathbf{z}_{p}}^{*}$;

(ii) if $p$ does divide $c_{i}$, then $\theta v_{i} \in \operatorname{Ker} i_{\mathbf{z}_{p}}^{*}$.

Let $I$ be $I=\left\{i ; p\right.$ does not divide $\left.c_{i}, m+2 \leqq i \leqq n\right\}$. Let $J=\{j ; j \notin I, m+1 \leqq j \leqq n\}$. Then $I=\left\{i ; b_{i}\right.$ is divided by the same power of $p$ as $\left.b_{i-1}\right\}$.

The important situation occurs when $i$ belongs to $J$. For example, $m+1$ and $N(p)$ are the smallest and the greatest elements of $J$.

We will change the generators of $H^{*}\left(Y_{n, n-m} ; \mathbf{Z}_{p}\right)$ to the following

then we obtain:

$$
\begin{aligned}
c_{i} x_{i} & =\theta v_{i}, & & i \in I, \\
x_{i} & =\bar{x}_{i}, & & i \in J, i \neq N(p) ;
\end{aligned}
$$

$$
H^{*}\left(Y_{n, n-m} ; \mathbf{Z}_{p}\right)=\mathbf{Z}_{p}[\bar{y}] /\left[\bar{y}^{N(p)}\right] \otimes \bigwedge\left(x_{m+1}, \ldots, \hat{x}_{N(p)}, \ldots, x_{n}\right)
$$

where

(7) $\theta v_{i}=c_{i} x_{i}, \quad i \in I ; \quad i_{\mathbf{Z}_{p}}^{*} x_{i}=\bar{z}_{i}, \quad m+1 \leqq i \leqq n, \quad i \neq N(p)$ and $\pi_{\mathbf{Z}_{p}}^{*}(\bar{\omega})=\bar{y}$.

Again in diagram (C), this time with $A=\mathrm{Q}$, we have

$$
\theta v_{i}=c_{i} \tilde{x}_{i}+\tilde{u}_{i}, \quad \tilde{u}_{i} \text { belongs to } \operatorname{Ker} i_{\mathbf{Q}}^{*}, \quad m+2 \leqq i \leqq n .
$$

We define $c_{i} w_{i}=\theta v_{i}, m+2 \leqq i \leqq n$ and we obtain

$$
\begin{aligned}
H^{*}\left(Y_{n, n-m} ; \mathbf{Q}\right)=\mathbf{Q}[\tilde{y}] /\left[\tilde{y}^{m+1}\right] \otimes & \wedge\left(w_{m+2}, \ldots, w_{n}\right) \\
& \text { where } \theta v_{i}=c_{i} w_{i}, \quad i_{\mathbf{Q}}^{*} w_{i}=\tilde{z}_{i}, \quad \pi_{\mathbf{Q}}^{*}(\tilde{\boldsymbol{\omega}})=\tilde{y} .
\end{aligned}
$$

3. Next, we compute the Bockstein spectral sequence of the couple

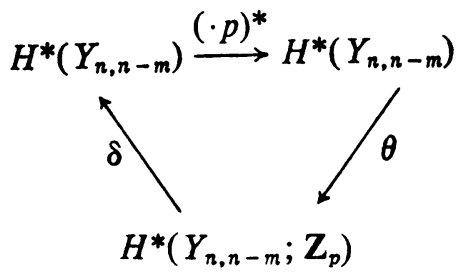

It follows from (7) that

$$
E_{1}=H^{*}\left(Y_{n, n-m} ; \mathbf{Z}_{p}\right)=\mathbf{Z}_{p}[y] /\left[y^{N}\right] \otimes \bigwedge\left(x_{m+1} \cdots \hat{x}_{N(p)} \cdots x_{n}\right)
$$

and from $\left(7^{\prime}\right)$ that

$$
E_{\infty}=H^{*}\left(Y_{n, n-m}\right) / \text { Torsion } \otimes \mathbf{Z}_{p}=\mathbf{Z}_{p}[y] /\left[y^{m+1}\right] \otimes \wedge\left(w_{m+2}, \ldots, w_{n}\right) .
$$


Recall that the differentials are Bockstein homomorphisms $\beta_{r}$ and an element $x \in E_{1}$, belongs to $\operatorname{Im} \theta$ if and only if

$$
\beta_{r} x=0 \text { for all } r \text {. }
$$

An element $y \in H^{*}\left(Y_{n, n-m}\right)$ has torsion $p^{r}$, that is $p^{r} a y=0$ where $p$ does not divide $a$, if and only if $\theta y \notin \operatorname{Im} \beta_{j}$ for $j<r$, but

$$
\theta y \in \operatorname{Im} \beta_{r} .
$$

First we will give some easy results:

If $x \in E_{r}$, call $\phi(x)$ its image in $E_{\infty}$, then

$$
\phi(\bar{y})=\tilde{y} ; \quad \phi\left(x_{i}\right)=w_{i}, \quad i \in I .
$$

By (7) and (8)

$$
\beta_{r}(\bar{y})=0, \quad \beta_{r}\left(x_{i}\right)=0, \quad \text { all } r, i \in I .
$$

By (10), since $w_{i} \neq 0$,

$$
x_{i} \notin \operatorname{Im} \beta_{r}, \quad \text { all } r, i \in I .
$$

We arrange $J$ so that $m+1=i(0)<i(1)<\cdots<i(j)<\cdots<i(t)=N(p)$ and put $b_{i(j)}=p^{r(j)} a_{j}$, where $p$ does not divide $a_{j}$; then $r(j)>r(j+1)$ and $b_{i}=p^{r(j)} a_{i}, i(j) \leqq i$ $<i(j+1)$.

By (9) and Proposition 5:

$$
y^{i} \notin \operatorname{Im} \beta_{r}, \quad r<r(j), \quad y^{i} \in \operatorname{Im} \beta_{r(j)}, \quad i(j) \leqq i<i(j+1) .
$$

Trivially

$$
E_{1}^{q}=E_{\infty}^{q}, \quad q<2 i(0)-1 .
$$

Now, we will compute $\beta_{r}$ :

LEMMA 8. The following formulae hold for every $j$

$$
\begin{gathered}
\beta_{r} x_{i(j)}=0, \quad r<r(j), \\
\beta_{r(j)} x_{i(j)}=k_{j} y^{i(j)}, \quad k_{j} \in \mathbf{z}_{p}, k_{j} \neq 0, \\
E_{1}^{q}=E_{r(j)}^{q}, \quad q<2 i(j+1)-1 .
\end{gathered}
$$

Proof. By (13) there is an element $x$ such that $\beta_{r(0,} x=y^{i(0)}$ but $x$ can only be a scalar multiple of $x_{i(0)}$ then (15) and (16) hold for $j=0$.

By the same argument (15) and (16) hold for $j=h$ provided that (17) holds for $j=h-1$.

In turn, (15) for every $j \leqq h$ and (11) together imply (17) for $j=h$ because Bockstein homomorphisms are derivations.

COROllary 9. For every $j$

$$
\begin{aligned}
\beta_{r(j)}\left(x_{i(j)} y^{s}\right)= & k_{j} y^{i(j)+s} \neq 0, \quad 0 \leqq s<i(j+1)-i(j), \\
& \beta_{r(j)}\left(x_{i(j)} y^{i(j+1)-i(j)}\right)=0 .
\end{aligned}
$$


Proof. (18) follows from (16) and (17). (19) follows from (16).

We call $u_{i(j+1)}$ the image of $x_{i(j)} y^{i(j+1)-i(j)}$ in $E_{r(j)+1}$.

It remains to prove that $\beta_{r}=0$ unless $r=r(j)$ for some $j$. This is part of the following lemma.

LEMMA 10. $\beta_{r}=0$ unless $r=r(j)$ and $E_{\infty}=E_{r}(0)$.

We use induction. Assign $\tilde{y}$ to $\bar{y}$ and $w_{i}$ to $x_{i}$ for $i \in I, i<i(1)$.

By $(15), \ldots,(19)$ and $\operatorname{dim} E_{\infty} \leqq E_{r(0)}$, this correspondence determines an isomorphism from $E_{\infty}$ onto $E_{r(0)}$, up to degree $2 i(1)-2$.

Moreover, $\beta_{r}=0$ up to degree $2 i(1)-2$ unless $r=r(0)$.

Suppose we have elements $\bar{u}_{i(j)}, j=1, \ldots, h$, such that:

(i) gr $\bar{u}_{i(j)}=2 i(j)-1$.

(ii) $\bar{u}_{i(j)} x_{i}=-x_{i} \bar{u}_{i(j)} ; \bar{u}_{i(j)} u_{i\left(j^{\prime}\right)}=-\bar{u}_{i\left(j^{\prime}\right)} \bar{u}_{i(j)}, j^{\prime}<j ;\left(\bar{u}_{i(j)}\right)^{2}=0$.

(iii) If we assign $\tilde{y}$ to $\bar{y} ; w_{i}$ to $x_{i}$ for $i \in I, i<i(j+1)$ and $w_{i(j)}$ to $\bar{u}_{i(j)}$ we determine an isomorphism from $E_{\infty}$ onto $E_{r(0)}$ up to degree $2 i(h+1)-2$.

Suppose besides that $\beta_{r}=0$ up to degree $2 i(h+1)-2$ unless $r=r(j), j=0, \ldots, t-1$.

From these assumptions and $(15), \ldots,(19)$ we have $\operatorname{dim} E_{r(0)}^{q}=\operatorname{dim} E_{\infty}^{q}, q \leqq$ $2 i(h+1)-1$ and all differentials are determined on all elements of degree $\leqq 2 i(h+1)$ -1 except $u_{i}(h+1)$ belonging to $E_{r(h)+1}$ and its images in $E_{r}, r>r(h)+1$.

Thus, for every $r, \beta_{r} u_{i(n+1)}$ must lie in the subspace of $E_{r}$ spanned by $\left\{\beta_{r} a\right\}$, where $a$ ranges over products. That means $\beta_{r} u_{i}(h+1)=0$ for $r<r(h-1)$; and there is an element $u^{\prime}$ in $E_{r(h-1)}^{2(h+1)-1}$, such that $\beta_{r(h-1)} u^{\prime}=0$ and $u^{\prime}$ does not belong to the subalgebra generated by elements with degree $<2 i(h+1)-1$. It is easy to see that $u^{\prime}$ satisfies (ii). Again, $\beta_{r} u^{\prime}=0$ for $r<r(h-2)$ and we repeat the argument until we reach $E_{r(0)}$, then we obtain an element $\bar{u}_{i(h+1)}$ in $E_{r(0)}$ to which we may assign $w_{i(h+1)}$. Now we assign $w_{i}$ to $x_{i}$ for $i \in I, i<i(h+2)$ and obtain an isomorphism up to degree $2 i(h+2)-1$. Then we have finished the proof of (i), (ii), (iii) with $j=h+1$. From (11) we see $\beta_{r}=0$ up to degree $2 i(h+2)-1$, unless $r=r(j)$ for $j=0, \ldots, t-1$. This completes the proof.

We have identified $H^{*}\left(Y_{n, n-m} ; \mathbf{Q}\right)$ with $E_{r(0)}$ as algebras, for every prime $p$. Then we have completed the proof of Theorem $A$.

\section{REFERENCES}

1. P. Baum and W. Browder, The cohomology of quotients of classical groups, Topology 3. (1965), 305-336.

2. A. Borel, Sur la cohomologie des espaces fibres principaux et des espaces homogenes des groupes de Lie compacts, Ann. of Math. 57 (1953), 115-207.

3. S. Gitler and D. Handel, The projective Stiefel manifolds. I, Topology 7 (1968), 39-46.

NORTHWESTERN UNIVERSTTY, Evanston, Illinois

UniversidAd DE BUENos Aires, Buenos Aires, Argentina 